\title{
Half-Full or Half-Empty: Does Gastroparesis Affect Lung Transplantation Outcomes?
}

\author{
Christopher Vélez $^{1} \cdot$ Kyle Staller $^{1}$
}

Accepted: 31 August 2021 / Published online: 15 September 2021

(c) The Author(s), under exclusive licence to Springer Science+Business Media, LLC, part of Springer Nature 2021

\section{Introduction}

Although significant advances in solid organ transplantation have been made in the past decades, outcomes inferior to those with other organ transplantations remain for lung transplantation [1]. Chronic lung allograft dysfunction (CLAD) remains the largest barrier to improved morbidity and mortality in lung transplant recipients [2]. Limiting effective management of CLAD is the multiplicity of pathophysiologic mechanisms that contribute to progressive transplant decline [3]. Any investigation into the factors that can be associated with CLAD is welcome; once such mechanism is gastroparesis. In this issue of Digestive Diseases and Sciences, Blackett et al.[4] make an important contribution to the understanding of the factors associated with the development of gastroparesis after lung transplantation. Using a single-center, retrospective design, they identified 616 patients who underwent lung transplantation at a large, diverse transplant center. More than 17\% (107) were diagnosed with gastroparesis within 3 years of transplant, with the most significant risk factor being Black race. Most interestingly, a new diagnosis of gastroparesis post-transplant was associated with an increased odds of CLAD, though there was no impact on overall mortality. Given inconsistent use of gastric emptying scintigraphy (GES, the most widely accepted means of diagnosing gastroparesis [5]) in this population, the current work raises the question as to whether GES should be considered as the newest addition to post-transplant protocols.

Gastroparesis is fundamentally a sensorimotor disorder provoked by a variety of processes. While the most common

Kyle Staller

kstaller@mgh.harvard.edu

1 Center for Neurointestinal Health, Division of Gastroenterology, Massachusetts General Hospital, Harvard Medical School, 15 Parkman Street, Suite 550F, Boston, MA 02114, USA etiology is "idiopathic," post-surgical interventions may result in gastroparesis - presumably via disruption of vagal nerve fibers [6]. Gastroparesis has been described after fundoplication for gastro-esophageal reflux as well as after solid organ transplantation, including lung transplantation. Whereas gastroparesis is typically thought of as a delay in gastric emptying (as defined by retention of $10 \%$ or more of tracer-labeled gastric contents at $4 \mathrm{~h}$ ), the severity of emptying delay does not necessarily correlate with symptoms, which makes singular use of the scintigraphy to define gastroparesis problematic [7].

Historically, management of gastroparesis has been difficult [6]. Pharmacologic targeting of motilin receptors (such as with erythromycin) or use of dopaminergic agents (such as metoclopramide or domperidone) can be limited by potentially irreversible side effects or medication-medication interactions that can be particularly problematic post-transplant. When pharmacologic management of gastroparesis failed, more invasive interventions were used cautiously, including surgical pyloroplasty or gastric electrical stimulation. Many patients were left unsatisfied and symptomatic. With newer effective and safer pharmacologic agents now available or in the pipeline and the evolution of per oral pyloroplasty, diagnosing gastroparesis is no longer merely an academic exercise. The current study suggests that transplant patients with gastroparesis may be missed despite potential benefits of treatment. Nevertheless, disorders of esophageal peristalsis and gastro-esophageal reflux disease (GERD) are likely still the major GI contributors to CLAD [8].

Blackett et al. have access to a robust single-center database of lung transplant patients that facilitates rigorous study of the factors associated with development of gastroparesis after lung transplantation. Their definition of incident GES as occurring within 3 years is a reasonable time frame, as undoubtedly confounders exist shortly after lung transplantation including the use of opiate analgesia, which may pharmacologically delay gastric emptying. Their study has some notable strengths including a large number of patients 
(175) having undergone GES and incorporation of multiple clinical, and demographic factors that could be associated with gastroparesis. The absence of an association between incident gastroparesis after lung transplantation and diabetes is surprising given the outsize contribution of diabetes to the understanding of the pathophysiology of gastroparesis. Similarly surprising is the lack of impact of single versus double lung transplant, since single lung transplant should in theory preserve at least one of the two vagal nerve trunks. One additional relevant finding from the current study is the link between gastroparesis and GERD (though GERD was not associated with CLAD in this cohort). It is increasingly recognized that these two entities may have a shared pathophysiology. Moreover, retained stomach contents in the setting of gastroparesis can increase the volume of esophageal refluxate.

There are, however, some notable weaknesses in this study. First and most importantly, most patients did not undergo diagnostic foregut testing. Only $28.4 \%$ of the patients underwent GES and $49.9 \%$ underwent ambulatory reflux testing. While the authors acknowledge this limitation, those undergoing diagnostic testing were presumably more likely to be symptomatic. Second, the lack of observed association between GERD and CLAD runs contrary to a widely accepted mechanism of CLAD both in transplant clinical practice and the literature. The reader should interpret the increased incidence of gastroparesis in Black patients as potentially confounded by other factors such as social determinants of health that warrant further study and may be influenced by healthcare resource usage. Finally, the results of any study relying on gastric emptying as a definitive endpoint should be interpreted with caution. Gastric emptying results are labile over time, with $>40 \%$ of patients with chronic upper GI symptoms and delayed gastric emptying normalizing over follow up despite ongoing symptoms [9]. It remains to be seen whether transplant patients with gastroparesis develop CLAD due to the pathophysiology of delayed emptying; alternatively, chronic upper GI symptoms may merely signify underlying neuromuscular dysfunction independent of gastric emptying status.

Despite these limitations, this study is an important first step in addressing an important question in post-lung transplant care: how to improve morbidity in mortality to the point that it approaches that of other solid organ transplants? Given the finite resource that are solid organ transplants, rigorous protocols are implemented at the institutional and national level in order to assure equitable allocation. Much care is taken in the peri-operative period and within the first year of transplantation to meet established benchmarks since these are fundamental measures by which transplant centers are appropriately judged. Yet, beyond this first year, there is more variability in the use of diagnostic testing to address the actionable physiologic mechanisms of gastroparesis and GERD that could potentially reduce the development of CLAD. Though the cost/effectiveness of systematic testing is debated, this must be weighed against the enormous investment made by healthcare systems in performing transplantations and in keeping transplant patients alive afterward. The association between CLAD and gastroparesis in the current work-should this association bear out in further study-is perhaps the strongest evidence yet suggesting transplant centers may need to be more liberal in physiologic testing after transplant surgery.

\section{Declarations}

Conflict of interest KS has received research support from AstraZeneca, Ironwood, and Urovant, has served as a speaker for Shire, and has served as a consultant to Arena, Gelesis, GI Supply, Synergy, and Shire. CV has no relevant disclosures.

\section{References}

1. Bos $\mathrm{S}$ et al. Survival in adult lung transplantation: where are we in 2020? Curr Opin Organ Transplant 2020;25:268-273.

2. Costa J, Benvenuto LJ, Sonett JR. Long-term outcomes and management of lung transplant recipients. Best Pract Res Clin Anaesthesiol 2017;31:285-297.

3. Verleden SE et al. Chronic lung allograft dysfunction phenotypes and treatment. J Thorac Dis 2017;9:2650-2659.

4. Blackett, J.W., et al., Risk factors and outcomes for gastroparesis after lung transplantation. Dig Dis Sci. (Epub ahead of print). https://doi.org/10.1007/s10620-021-07249-y.

5. Camilleri M et al. Gastroparesis. Nat Rev Dis Primers 2018;4:41.

6. Vélez C, Kuo B. Gastroparesis and the brain-gut axis. In: McCallum R, Parkman H, eds. Cambridge: Academic Press; 2021; 95-107.

7. Grover M, Farrugia G, Stanghellini V. Gastroparesis: a turning point in understanding and treatment. Gut 2019;68:2238-2250.

8. Hathorn KE, Chan WW, Lo WK. Role of gastroesophageal reflux disease in lung transplantation. World J Transplant 2017;7:103-116.

9. Pasricha PJ et al. Functional dyspepsia and gastroparesis in tertiary care are interchangeable syndromes with common clinical and pathologic features. Gastroenterology 2021;160:2006-2017.

Publisher's Note Springer Nature remains neutral with regard to jurisdictional claims in published maps and institutional affiliations. 